\title{
Use of an autologous platelet therapy in police working dogs with hip osteoarthritis
}

\section{Alves J.1,2, *; Santos A. ' ; Jorge P. '; Lavrador C. ${ }^{2}$; Carreira L. Miguel 3,4}

'Guarda Nacional Republicana; 'DIMED - Mediterranean Institute for Agriculture, Environment and Development, Universidade de Évora; ${ }^{3}$ Interdisciplinary Centre for Research in Animal Health (CIISA) - University of Lisbon, (FMV/ULisboa) - Portugal. ${ }^{4}$ Anjos of Assis Veterinary Medicine Centre; *alves.jca@gnr.pt

\section{OPBJECLIIVIES: \\ To describe the effect of the platelet concentrate V-PET in dogs with hip osteoarthritis (OA).}

\section{MATERIALS AND METHODS:}

Forty joints of active police working dogs with hip OA were randomly assigned to control and V-PET groups;

Weight bearing distribution, joint range of motion, thigh girth, radiographic signs, and four clinical metrology instruments (CMI) were recorded at treatment day, 8, 15, 30, 90, and 180-days post-treatment;

Results were compared with repeated measures ANOVA, with a Huynh-Feldt correction, or Wilcoxon Signed Ranks Test, with $p<0.05$. Kaplan-Meier estimators were conducted and compared with the log-rank test.

\section{RESULTS:}

Patients had a mean age of $6.5 \pm 2.4$ years and bodyweight of $26.7 \pm 5.2 \mathrm{~kg}$. At T0, 32 (80\%) joints were graded as mild, 6 (15\%) as moderate, and 2 (5\%) as severe OA. No differences were found between groups on treatment day.

Weight-bearing showed significant improvements with V-PET from 8 days $(p<0.01)$ up to 180 days $(p=0.01)$, as joint flexion up to 90 days $(p=0.05)$, and extension up to 180 days $(p=0.01)$.

Several CMI scores improved up to 90 to 180 days post-treatment.

Kaplan-Meier estimators showed significantly better CMI and weight-bearing results in the V-PET group (Figures)
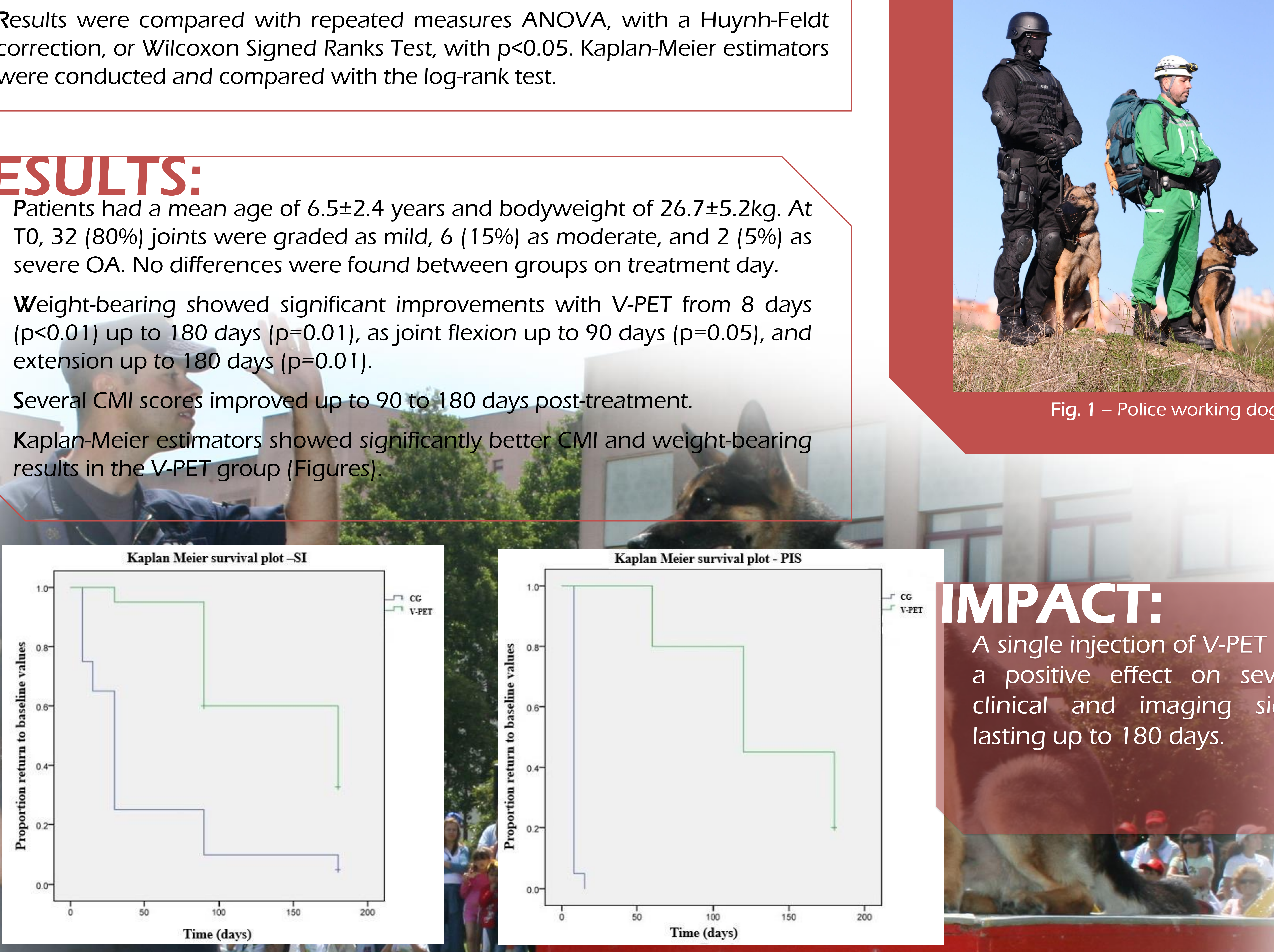

Fig. 1 - Police working dogs. 